\title{
ENERGY DISTRIBUTIONS OF SYMBIOTIC NOVAE
}

\author{
Greg L. BRYAN \\ Department of Physics and Astronomy, University of Calgary \\ AND \\ SUN KwOK ${ }^{1}$ \\ Joint Institute for Laboratory Astrophysics, National Institute of Standards and Technology and University of Colorado \\ Received 1990 April 19; accepted 1990 August 6
}

\begin{abstract}
The IRAS low-resolution spectra of three recent symbiotic novae (HM Sge. V1016 Cyg, and RR Tel) are fitted with a dust continuum radiative transfer model. We find that the dust shells are detached from the photosphere and that the sizes of the inner radii are correlated with times since outburst. An analysis of the IUE spectra of HM Sge at different epochs suggests that the strength of the $2200 \AA$ feature is decreasing with time and the grains responsible for the feature are probably formed in the white dwarf ejecta. A complete accounting of the entire energy budget from radio to X-ray shows that most of the energy is emitted by the cool component in the infrared, and a significant fraction of the flux of the hot component is escaping in the far-ultraviolet. The density-bounded nature of the circumstellar gas nebulae could be the result of a bipolar geometry of the nebulae. Unlike classical novae, the optical outburst of symbiotic novae is due to the ionization of the preexisting envelope of the cool component and is not the result of a sudden ejection by the hot component.
\end{abstract}

Subject headings: infrared: spectra - stars: circumstellar shells - stars: symbiotic - ultraviolet: spectra

\section{INTRODUCTION}

Symbiotic novae belong to a class of objects that show a simultaneous presence of nebular and cool star spectral characteristics and that have had a large outburst in the visible in the recent past (Allen 1980). They are generally understood as binary systems consisting of a cool Mira variable (Whitelock 1987, 1988) and a hot white dwarf (Kenyon and Truran 1983). The circumstellar nebular material is provided by the wind of the Mira component and the hot star is responsible for the ionization of the nebula, leading to an emission-line spectrum. It is also widely believed that the optical outburst is the result of the ignition of the nuclear burning on the surface of a white dwarf, causing the star to evolve (backward) to an active state similar to that of a central star of planetary nebula (Paczyński and Rudak 1980; Kenyon and Truran 1983).

Symbiotic novae are interesting objects to study because they are active in almost every part of the electromagnetic spectrum. In the visible and near-ultraviolet, the spectrum is dominated by emission from the ionized nebula, with most of the energy emitted in recombination lines of hydrogen and helium as well as forbidden lines of heavy elements. The continuum is made up of bound-free radiation. In the nearinfrared, the photosphere of the Mira variable can often be seen (cf. Whitelock 1987), although highly reddened by circumstellar dust (Kenyon, Fernandez-Castro, and Stencel 1988). The dust continuum dominates the radiation between 10 and $100 \mu \mathrm{m}$. At longer wavelengths $(\lambda \sim 1 \mathrm{~mm})$, the nebular freefree continuum exceeds the dust continuum (cf. Kwok 1982). Symbiotic novae are also found to be strong X-ray emitters (Allen 1981). The X-ray spectrum is believed to be due to thermal bremsstrahlung, although the spectral shape is not accurately known due to the effects of interstellar absorption.

1 JILA Visiting Fellow 1989-1990, on leave from University of Calgary, Calgary, Alberta, Canada.
In this paper, we have analyzed the mid-infrared spectra of three symbiotic novae using data from the Low Resolution Spectrometer (LRS) of the Infrared Astronomical Satellite (IRAS). The IRAS observations have provided excellent quality spectra of these objects, particularly in the region 13-23 $\mu \mathrm{m}$ which is not observable from the ground. Data in the International Ultraviolet Explorer (IUE) archive on these objects have also been analyzed to obtain estimates of the continuum. All the data in the presently observable electromagnetic spectrum are combined in an effect to understand the energetics of these objects.

\section{IRAS LOW-RESOLUTION SPECTRAL DATA}

LRS was one of the survey instruments on the IRAS satellite. It was in operation during the entire mission to measure the spectra of bright point sources between 7.7 and $22.6 \mu \mathrm{m}$ with a resolution varying from 60 on the short-wavelength side to 20 on the long-wavelength side. Whenever the survey detectors indicated that a point like source brighter than $2 \mathrm{Jy}$ at 12 or $25 \mu \mathrm{m}$ had crossed the aperture, the corresponding sections of the LRS data stream were extracted. Two overlapping wavelength bands were scanned simultaneously, one ranging from 7.7 to $13.4 \mu \mathrm{m}$ and the other from 11.0 to $22.6 \mu \mathrm{m}$. The scans corresponding to HM Sge, V1016 Cyg, and RR Tel were extracted from the LRS data base, and a weighted average spectrum was obtained for each object. A weighting factor was assigned for each scan, inversely proportional to the square of the rms noise, which in turn was determined by the fluxes in the first and last 20 channels of each band. Spectra obviously contaminated by spurious results were not included. A linear instrumental baseline was removed, its parameters again determined in the channels without data. The two bands were joined to ensure continuity by constructing linear fits over the overlap region (11-13 $\mu \mathrm{m})$ and the entire spectrum was convolved with the $12 \mu \mathrm{m} I R A S$ instrumental profile and normalized to the $12 \mu \mathrm{m}$ broad-band in-band flux. The spectrum was 


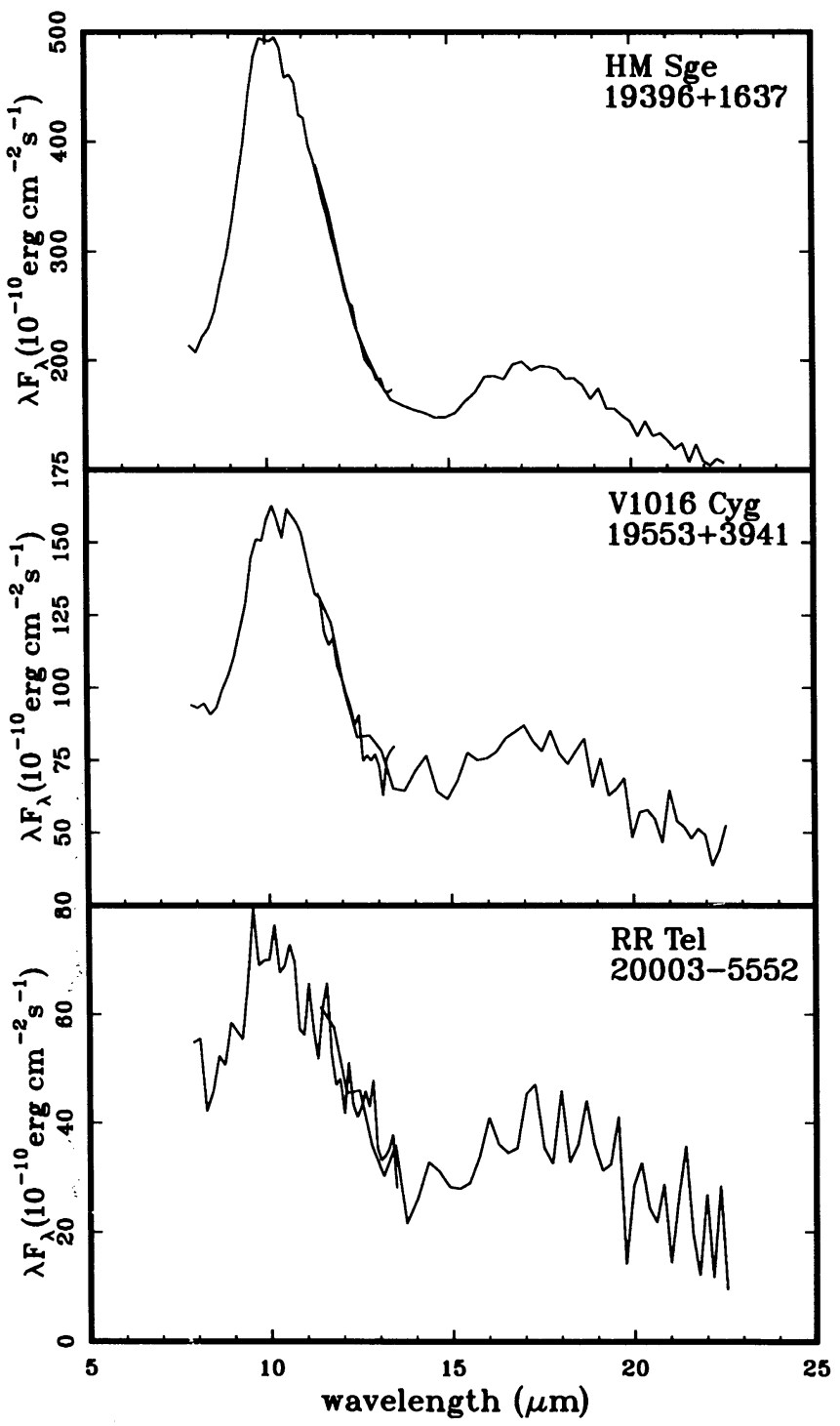

FIG. 1.-The LRS spectra of (top) HM Sge, (middle) V1016 Cyg, and (bottom) RR Tel. The IRAS names are listed below the variable star names.

also recalibrated using the procedure of Volk and Cohen (1989). The processed LRS spectra of HM Sge, V1016 Cyg, and RR Tel are shown in Figure 1.

The LRS spectra of all three stars show strong silicate emission features at 9.7 and $18 \mu \mathrm{m}$, suggesting that the cool component is a mass-losing Mira variable. This confirms that the mid- and far-infrared continua of these three objects are circumstellar in origin, and not just due to highly reddened photospheric continua as suggested by Kenyon, FernandezCastro, and Stencel (1986). In Figures $2 a, 2 b$, and $2 c$ we have plotted the LRS spectra of HM Sge, V1016 Cyg, and RR Tel together with the $12,25,60$, and $100 \mu \mathrm{m} I R A S$ broad-band fluxes (Kenyon, Fernandez-Castro, and Stencel 1986) after correction for color effects. We have also collected photometric measurements in the UBVRIJHKLM bands. Measurements at the same epochs are connected by dashed lines in Figure 2. These measurements (although not complete) clearly indicate that the objects are variable. The pulsation periods of the Mira components are estimated to be 540,450 , and 387 days for HM Sge, V1016 Cyg and RR Tel, respectively (Whitelock 1988).

In the near-infrared, the detection of molecular absorption bands suggests that the observed fluxes are dominated by the reddened photospheric continua of the Mira components (Puetter et al. 1978). In the visible region, we begin to see excesses above the cool star's photospheric emission. Since emission lines contributed strongly to the optical photometry, estimates of the continuum levels are also indicated in Figure 2. The data for HM Sge are taken from Davidson, Humphreys, and Merrill (1978), Taranova and Yudin (1983), Mueller and Nussbaumer (1985), Feibelman (1982), and Blair et al. (1981); whereas data for V1016 Cyg are from Ahern (1978), Swings (1981), Feibelman (1982), and Nussbaumer and Schild (1981). The level of the continuum of $\mathbf{R R}$ Tel is estimated from Sahade, Brandi, and Fontenla (1984) and Penston et al. (1983).

The difficulty of obtaining continuum information on these three objects is illustrated by the widely varying values obtained in the literature (Fig. 2) and can be understood by the relatively low flux level of the continuum. There are many estimates on the continuum level of HM Sge. While there is no consensus on the absolute level, it is generally agreed that it is very flat in $F_{\lambda}$, possibly rising at longer wavelengths (Ciatti, Mammano, and Vittone 1978). a fairly strong Balmer discontinuity is reported (Arkhipova and Dokuchaeva 1978), indicating that bound-free radiation is important. A similar situation exists with V1016 Cyg, except that the continuum is probably rising with wavelength (Nussbaumer and Schild 1981). The Balmer discontinuity in the continuum was noted by O'Dell (1967), Baratta, Cassatella, and Viotti (1974), and Ahern (1978). Swings (1981) provides data to $1 \mu \mathrm{m}$, showing the very sharp rise of the cool star's photosphere. Finally, RR Tel appears to have a brighter UV continuum which is decreasing in $F_{\lambda}$ with increasing wavelength. This object too, shows a significant Balmer discontinuity (Penston et al. 1983).

\section{MODEL OF THE DUST CONTINUUM EMISSION}

Although symbiotic novae are binary systems, the dust envelope is probably produced by the stellar wind from the Mira component (Kwok 1982). In fact, the infrared spectra of these symbiotic novae are very similar to other mass-losing Mira variables on the asymptotic giant branch (AGB) (Volk and Kwok 1987). Since the size of the circumstellar envelope $(\sim 1 \mathrm{pc})$ is also much greater than the binary separation $(\sim 10$ $100 \mathrm{AU})$, the circumstellar dust emission can be reasonably approximated by a spherically symmetric system. One can also assume that the dust is primarily heated by the cool star. This can be justified in the following way. Since the bolometric luminosities of the hot and cool components are probably comparable, the high temperature $\left(\sim 10^{5} \mathrm{~K}\right)$ of the hot component means that the cool stars have far more photons available for heating of the dust. For photons shortward of the Lyman continuum, the gas nebula is a strong competitor and is more efficient in absorbing the UV photons than the dust.

Under these assumptions, we can model the infrared spectra of these symbiotic novae with a radiative transfer program. The DUSTCD code of Leung $(1975,1976)$ is used with the silicate grain opacity function of Volk and Kwok (1988). An inverse-square density profile is divided into 161 radial grid points arranged logarithmically. The wavelength range of 0.1 $\mu \mathrm{m}$ to $1 \mathrm{~cm}$ is divided into a 150 grid points, with denser distributions around the dust features where the emission peaks. The source function and the emergent spectrum are 

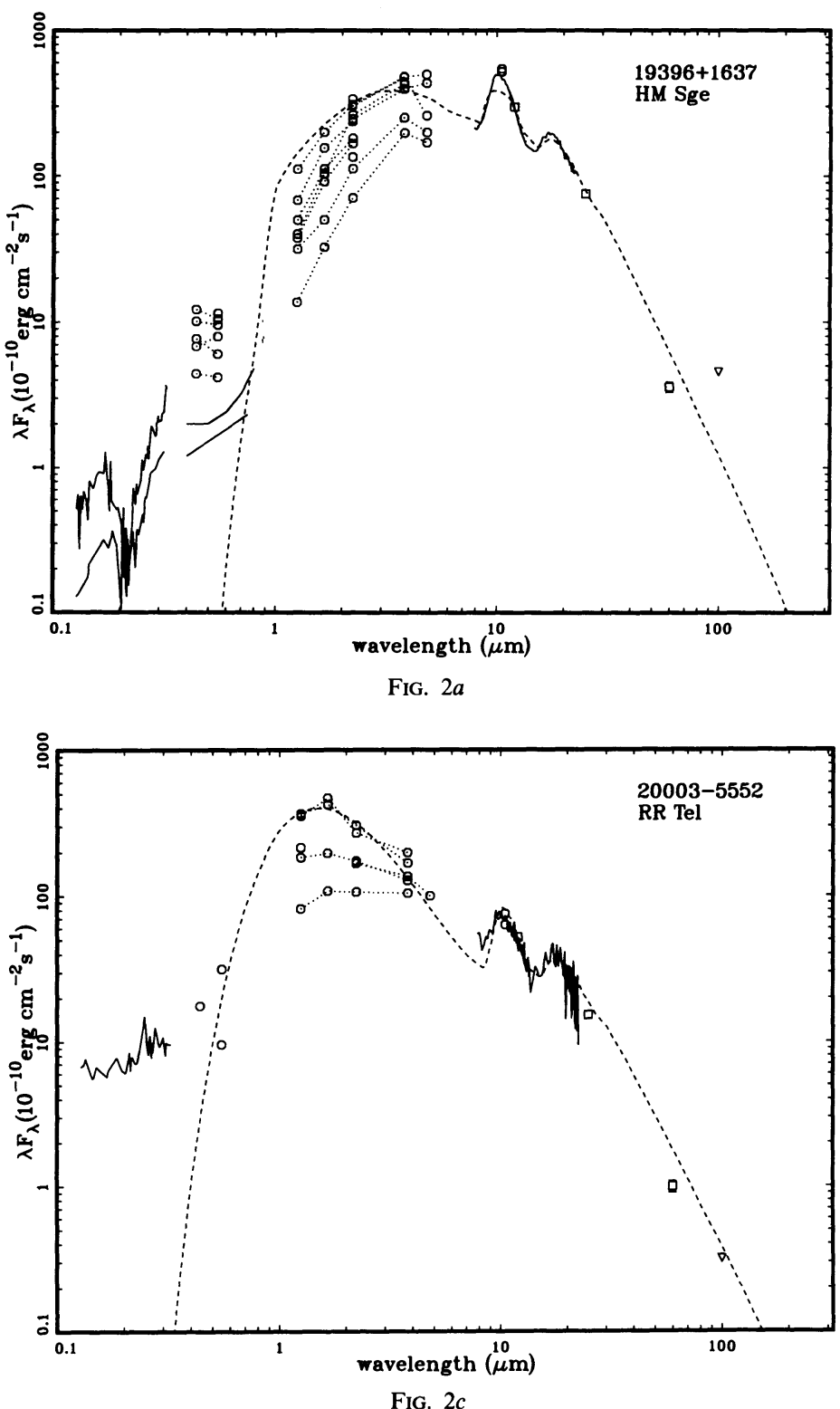

FIG. 2.-Model fits to the circumstellar dust emission of $(a)$ HM Sge, $(b)$ V1016 Cyg, and (c) RR Tel. The circles are ground-based optical and nearinfrared photometric measurements not corrected for reddening. Observations of the same epoch are joined by dotted lines. The optical photometry is made up of broad-band measurements including contributions from emission lines. The LRS spectra (solid line in the 8 to $23 \mu \mathrm{m}$ region) have been convolved with the $12 \mu \mathrm{m}$ instrumental profile and normalized to the $12 \mu \mathrm{m}$ point. Colorcorrelated IRAS photometry is shown as open squares. The estimated levels of the ultraviolet continuum are shown as solid lines between 1000 and $3000 \AA$. The solid lines between 5000 and $8000 \AA$ in HM Sge and V1016 Cyg are estimates of the continuum levels by Blair et al. (1981) and Taranova and Yudin (1983) (HM Sge) and Swings (1981) and Ahern (1978) (V1016 Cyg). The dashed line is the emergent spectrum predicted by the dust continuum radiative transfer model.

determined interactively. The effective temperatures of the cool components are assumed to be $3000 \mathrm{~K}(2500 \mathrm{~K}$ for RR Tel), typical of late-type Mira variables with periods of 400-600 days. However, a change in effective temperature of $\pm 500 \mathrm{~K}$ has little effect on the model results.

In spite of the superficial resemblance of the infrared spectra to those of AGB stars, results of the model fitting indicate one

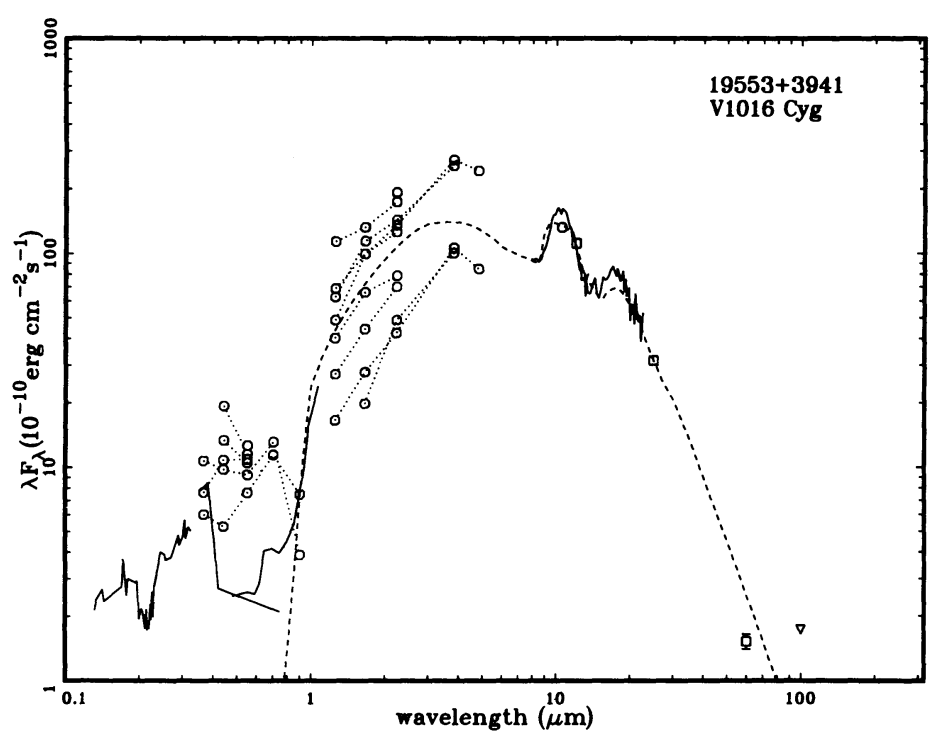

FIG. $2 b$

major difference between symbiotic novae and AGB stars. The $10-100 \mu \mathrm{m}$ fluxes of the sources require a large amount of dust in the envelope (or equivalently a high mass-loss rate), and yet the photosphere is clearly visible. This can be accomplished only by having the inner radius of the dust envelope well separated from the photosphere, in contrast to AGB stars. Table 1 shows the results of the best-fit parameters for the three systems.

Assuming a typical bolometric luminosity of $6000 \mathrm{~L}_{\odot}$ for Mira variables of periods $\sim 400^{\mathrm{d}}$ (Feast and Whitelock 1987), we see that the derived inner radii corespond to $10.5 R_{*}$ for $\mathrm{HM}$ Sge, $18 R_{*}$ for V1016 Cyg, and $58 R_{*}$ for RR Tel. These ratios will be even higher if the effective temperatures are higher than $2500 \mathrm{~K}$. From the derived total fluxes and the assumed luminosities of $6000 L_{\odot}$, we can estimate that the distances to the objects are 1.4, 2.4, and $1.8 \mathrm{kpc}$ for HM Sge, V1016 Cyg, and RR Tel, respectively.

Comments on the fittings of the individual objects are as follows.

1. HM Sge.-The fitting of this object requires a delicate balance between reproducing the strength of the 9.7 and $18 \mu \mathrm{m}$ silicate emission features and the level of the reddened photosphere. Our best efforts predict a $9.7 \mu \mathrm{m}$ feature that is weaker than observed and a photospheric component brighter than observed. The value of circumstellar extinction when extrapolated to the visible $\left(A_{v}\right)$ is 8.7 . The agreement between the model and observations will improve if interstellar extinction is included. Our model fittings show that the model parameters are rightly constrained by the observations. The uncertainty in the optical depth is very small, given the requirements discussed above. The value of the inner radius is constrained by the need to produce a large amount of flux in the mid-infrared and a small amount of extinction in the near-infrared. The uncertainties in the model parameters would be larger if the hot star contributes a substantial amount of heating to the dust component.

2. V1016 Cyg.-A reasonable fit to the data was achieved with only minor problems. The photosphere is sufficiently reddened $\left(A_{v}=9.7\right)$ but there are excesses at the $L$ and $M$ bands, causing the photosphere to assume a sharp, straight rise 
TABLE 1

Dust Continuum Transfer Model Parameters

\begin{tabular}{cccccc}
\hline Object & $\begin{array}{c}T^{*} \\
(\mathrm{~K})\end{array}$ & $\begin{array}{c}D\left(L_{*} / 6000 L_{\odot}\right)^{1 / 2} \\
(\mathrm{kpc})\end{array}$ & $\begin{array}{c}R_{\mathrm{in}} / D \\
\tau(9.7 \mu \mathrm{m})\end{array}$ & $\begin{array}{c}\dot{M} / D / V \\
\left(10^{14} \mathrm{~cm} \mathrm{kpc}^{-1}\right)\end{array}$ & \begin{tabular}{c}
{$\left[M_{\odot} \mathrm{yr}^{-1} \mathrm{kpc}^{-1}\left(\mathrm{~km} \mathrm{~s}^{-1}\right)^{-1}\right]$} \\
\hline HM Sge $\ldots \ldots \ldots \ldots \ldots \ldots$
\end{tabular} \\
V1016 Cyg $\ldots \ldots \ldots \ldots \ldots$ & 3000 & 1.4 & 1.8 & 1.5 & $3.0(-7)$ \\
RR Tel $\ldots \ldots \ldots \ldots \ldots \ldots$ & 2500 & 1.8 & 2.0 & 1.5 & $2.7(-7)$ \\
\hline
\end{tabular}

to $4 \mu \mathrm{m}$. Again, the predicted 9.7 and $18 \mu \mathrm{m}$ dust features are weaker than observed.

3. RR Tel.-This model (Fig. 2c) provides an excellent fit to the most luminous phase of the Mira (except for a small excess in $L$ band). It is interesting to note that the dimmer phases captured in the broad-band photometry show flatter and flatter peaks. This can be reproduced with a higher optical depth and smaller inner radius (although the $B$ and $V$ points no longer fit well; this could be due to emission lines in these visible bandpasses). The model estimated extinction coefficient $\left(A_{v}\right)$ has a value of 0.5 which is significantly less than HM Sge and V1016 Cyg. This is probably a result of the dispersion of the dust envelope as the result of expansion. The smaller amount of circumstellar extinction is also responsible for the significant contribution of the $M$ star photosphere to the visible continuum.

While the above modeling process can yield an accurate estimate of the optical depth in the mid-infrared wavelengths, the estimates of visual extinction is based on an assumed opacity function of the silicate grain in the visible, and could be subject to error. Nevertheless, the large optical depths at $10 \mu \mathrm{m}$ for HM Sge and V1016 Cyg suggest that circumstellar extinction in the visible should still be substantial. For comparison, Thronson and Harvey (1981) derive a value for $A_{v}$ of $12 \pm 1$ from the Brackett line ratios and case $B$ recombination coefficients, whereas the Balmer line ratios give values of $A_{v} \sim 1$ (Davidson, Humphreys, and Merrill 1978; Blair et al. 1981; Stauffer 1984).

One notes that the derived inner radius of the circumstellar envelope is largest in RR Tel and smallest in HM Sge. This is in agreement with the expectation that older symbiotic novae have been subjected to the "snowplow" effect for a longer period of time (Kwok 1988), and therefore a larger inner radius.

While the size of the dust envelope is much larger than the binary separation, the derived inner radii of the three systems are comparable to the orbital size. As a result, the degree of heating contributed by the hot component will be dependent on the orbital phase of the system. The amount of visual extinction to the hot component will also vary with phase. This possibility was considered by Kenyon, Fernandez-Castro, and Stencel (1986), as well as by Allen (1983) for the symbiotic system $\mathrm{H} 1-36$.

Using 13 flux values as approximations to the 200 channel LRS spectrum, Anandarao, Taylor, and Pottasch (1988) fitted the LRS and photometry with two dust shells plus the cool star. Altogether six parameters (optical depth, temperature, and angular sizes of the two dust shells) were used, in addition to an unspecified temperature of the cool star and the dust opacity function. Their expression for the two-shell model (see their eq. [1]) is true only under optically thin conditions, but some of the optical depth values they derived are greater than unity. However, the two-shell model can be viewed as a crude approximation to the density structure of the circumstellar envelope. Anandarao, Taylor, and Pottasch (1988) also stated that the dust is heated by the hot star, but no energy balance calculation was performed to support the statement. In spite of the crudeness of the data and model used, they nevertheless arrived at the qualitative result that the temperatures of the dust shells decrease with the time since outburst, a conclusion supported by the more detailed calculation presented here.

\section{DATA FROM IUE}

Many observations from the IUE have been made for these objects. We have reanalyzed a number of these observations using the IUE Regional Data and Analysis Facility at the University of Colorado. Table 2 lists the images used; they are all at low dispersion with the large aperture. After extraction, the images were reduced with the IUELO reduction package, making corrections for the camera temperature and sensitivity adjustments for LWR images. The images for each source were

TABLE 2

IUE IMAGES USED IN UV CONTINUUM DETERMINATION

\begin{tabular}{|c|c|c|}
\hline Image & $\begin{array}{l}\text { Exposure Time } \\
\text { (minutes) }\end{array}$ & $\begin{array}{c}\text { Date of } \\
\text { Observations }\end{array}$ \\
\hline \multicolumn{3}{|c|}{ HM Sge } \\
\hline LWP 8879 & 10 & 1986 Aug 14 \\
\hline LWP 8880 & 90 & 1986 Aug 14 \\
\hline LWP 10478 & 10 & 1987 Apr 3 \\
\hline LWP $10479 \ldots$ & 20 & 1987 Apr 3 \\
\hline LWR $4675 \ldots$ & 30 & 1979 Jun 2 \\
\hline LWR $6960 \ldots$ & 30 & $1980 \mathrm{Feb} 20$ \\
\hline LWR $11871 \ldots$ & 30 & 1981 Oct 31 \\
\hline LWR 13013 .. & 60 & 1982 Apr 13 \\
\hline SWP $5430 \ldots$ & 60 & 1979 Jun 2 \\
\hline SWP $16705 \ldots \ldots \ldots$ & 10 & 1982 Apr 6 \\
\hline SWP $16706 \ldots$ & 10 & 1986 Apr 6 \\
\hline SWP $28897 \ldots$ & 100 & 1986 Aug 14 \\
\hline SWP 30694 & 92 & 1987 Apr 3 \\
\hline \multicolumn{3}{|c|}{ V1016 Cyg } \\
\hline LWR 2229 & 40 & 1978 Aug 31 \\
\hline LWP 4959 & 25 & 1984 Dec 11 \\
\hline LWR 6227 . & 15 & 1979 Nov 25 \\
\hline LWR $15597 \ldots \ldots \ldots \ldots \ldots$ & 25 & 1983 Mar 28 \\
\hline SWP $2427 \ldots \ldots \ldots \ldots \ldots$ & 40 & 1978 Aug 31 \\
\hline \multicolumn{3}{|c|}{ RR Tel } \\
\hline LWP $8179 \ldots \ldots \ldots \ldots \ldots$ & 3 & 1986 May 12 \\
\hline LWP $8726 \ldots \ldots \ldots \ldots \ldots$ & 2 & $1986 \mathrm{Jul} 24$ \\
\hline LWP 10918. & 2 & 1987 Jun 5 \\
\hline LWR 9239 ................ & 3 & 1980 Nov 5 \\
\hline LWR $16186 \ldots \ldots \ldots \ldots \ldots$ & 2 & 1983 Jun 18 \\
\hline SWP $10553 \ldots \ldots \ldots \ldots \ldots$ & 0.5 & 1980 Nov 5 \\
\hline SWP $28743 \ldots \ldots \ldots \ldots \ldots$ & 2 & 1986 July 24 \\
\hline SWP $29536 \ldots \ldots \ldots \ldots \ldots$ & 3 & 1986 Oct 26 \\
\hline SWP $29537 \ldots \ldots \ldots \ldots \ldots$ & 10 & 1986 Oct 26 \\
\hline
\end{tabular}


combined to realize a high signal-to-noise ratio after first ensuring that the continua did not appear to vary between exposures (with the exception of HM Sge, see below). Each image was resampled onto the same wavelength grid and weighted according to the square root of the exposure time. The long- and short-wavelength portions for each object were joined at $1975 \AA$, and only data in the range $1250-3250 \AA$ were used.

In an effort to determine the continuum levels from the $I U E$ images, we have averaged the spectra in $20 \AA$ bins in regions that appeared line-free. The results are shown in Figure 3. The $2200 \AA$ absorption feature is clearly visible in HM Sge and V1016 Cyg and is probably also present in RR Tel. Assuming the extinction law of Savage and Mathis (1979), we have used this feature to determine the amount of extinction by trying various $E(B-V)$ values until the feature disappears. This procedure is similar to that used by Mueller and Nussbaumer (1985) for HM Sge, for example. The dereddened continua are shown on the right side in Figure 3 and the $E(B-V)$ values in Table 3.

The images of HM Sge, when their continua are compared,
TABLE 3

EXTINCTION COEFFICIENTS FROM THE $2200 \AA$ FeATURE

\begin{tabular}{|c|c|}
\hline Object & $E(B-V)$ \\
\hline HM Sge (early) & $0.70 \pm 0.1$ \\
\hline HM Sge (late) ......... & $0.45 \pm 0.1$ \\
\hline 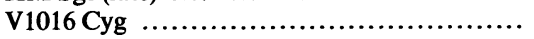 & $0.30 \pm 0.1$ \\
\hline RR Tel $\ldots \ldots \ldots \ldots \ldots \ldots \ldots \ldots \ldots \ldots \ldots \ldots \ldots \ldots \ldots \ldots \ldots \ldots \ldots$ & $0.10 \pm 0.05$ \\
\hline
\end{tabular}

fall into two readily identifiable groups with relatively little variation within the group. We will refer to the images taken in 1979-1982 as the "early" group, and those taken in 1986-1987 as the "late" group. We note that the early spectra have a much stronger $2200 \AA$ feature, resulting in an $E(B-V)$ difference of 0.4 between the two epochs. This suggests that the cause of the $2200 \AA$ feature is circumstellar and not interstellar. The grains responsible for the $2200 \AA$ feature are unlikely to be from the $M$ giant because the cool component is oxygen-rich, whereas the $2200 \AA$ feature is believed to be due to carbonbased material. It is probably formed in the ejecta from the
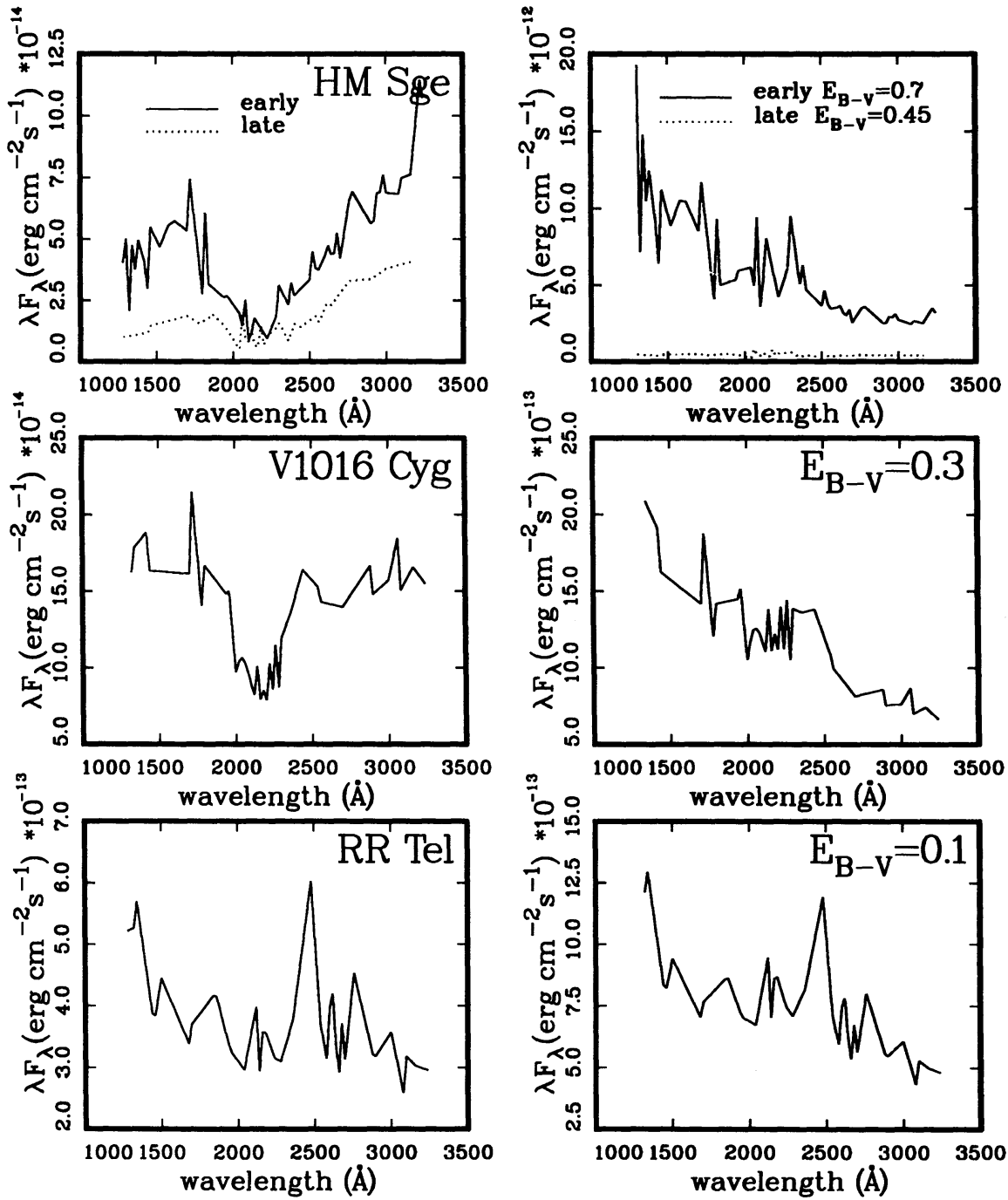

Fig. 3. - The derived IUE continuum for HM Sge, V1016 Cyg, and RR Tel. The three spectra on the right have been dereddened and the derived $E(B-V)$ values are shown on the upper right corner. See text for details. 
white dwarf component, and the extinction has decreased as the result of expansion. There are also indications of a similar decrease in extinction as estimated from the Balmer lines. The value of $E(B-V)$ changes from 0.8 in 1977 (Davidson, Humphreys, and Merrill 1978) to 0.4 in 1981 (Blair et al. 1981). It should be noted that the $E(B-V)$ values derived here are only the extinction due to the white dwarf ejecta and represent only part of the total amount of circumstellar and interstellar extinction.

The shapes of the continua from the two epochs are also different. The dereddened continuum is almost flat in the late images, but the early continuum is consistent with a RayleighJeans tail of $\sim 40,000 \mathrm{~K}$. This could be due to the psuedophotosphere of the ejecta from the white dwarf, and the subsequent decline in flux level could be the result of the contraction of the psuedophotosphere as the ejection diminishes. This is consistent with the disappearance of the W-R feature in the optical spectrum of HM Sge after 1978 (Feibelman 1982; Stauffer 1984). A continuous change of the continuum of HM Sge between 1978 and 1989 was also noted by Nussbaumer and Vogel (1990). They did not, however, note any change in the reddening coefficient. Using both the $2200 \AA$ feature as well as the He II lines they derived an average $E(B-V)$ of $0.6 \pm 0.1$ in the period 1978-1989.

\section{ENERGY DISTRIBUTION OF SYMBIOTIC NOVAE}

In order to consider the overall energy budget of symbiotic novae, we have plotted in Figure 4 the observed energy distributions between $100 \AA$ and $10 \mathrm{~cm}$ for the three objects. The radio data for $\mathrm{HM}$ Sge were taken from Kwok, Bignell, and Purton (1984), whereas the compiled data from Purton et al. (1982) were used for V1016 Cyg and RR Tel.

Also plotted is a theoretical curve corresponding to the expected free-free emission from a stellar wind (Wright and Barlow 1975; Olnon 1975). The inner radii derived from the dust model (cf. $\S$ III) are used in the fit. These inner radii predict that the spectra turn over at $\sim 100 \mathrm{GHz}$, and the extrapolated level of optically thin fluxes are consistent with the $100 \mu \mathrm{m}$ upper limits observed by $I R A S$. The derived massloss rates $(\dot{M} / V) D^{-2}$ are $2.4 \times 10^{-7}, 1.9 \times 10^{-7}$, and $3.2 \times 10^{-8} M_{\odot} \mathrm{yr}^{-1}$ for HM Sge, V1016 Cyg, and RR Tel, respectively.

In the infrared, we have plotted the IRAS measurements after color correction. The eight mid-infrared photometry points for HM Sge were obtained at the Canada-FranceHawaii Telescope on 1984 May 14. The derived magnitudes are summarized in Table 4.

In the visible region, we have plotted in Figure $4 a$ the emis-

TABLE 4

INFRared PhOtometry of HM SAgitTae

\begin{tabular}{|c|c|c|}
\hline $\begin{array}{c}\text { Central } \\
\text { Wavelength } \\
(\mu \mathrm{m})\end{array}$ & $\begin{array}{c}\text { FWHM } \\
(\mu \mathrm{m})\end{array}$ & Magnitude \\
\hline $7.8 \ldots$ & 0.7 & $0.70 \pm 0.05$ \\
\hline $8.7 \ldots$ & 1.2 & $-0.25 \pm 0.05$ \\
\hline $9.8 \ldots \ldots \ldots \ldots \ldots \ldots$ & 1.2 & $-1.21 \pm 0.05$ \\
\hline $10.3 \ldots \ldots \ldots \ldots \ldots \ldots \ldots$ & 1.0 & $-1.36 \pm 0.05$ \\
\hline '............... & 1.3 & $-1.44 \pm 0.05$ \\
\hline $12.5 \ldots \ldots \ldots$ & 1.2 & $-0.84 \pm 0.10$ \\
\hline $20.0 \ldots \ldots \ldots$ & 9 & $-2.67 \pm 0.10$ \\
\hline $25.0 \ldots \ldots \ldots \ldots \ldots \ldots \ldots$ & 10 & $-2.93 \pm 0.20$ \\
\hline
\end{tabular}

sion lines observed by Blair et al. (1981). The line intensities were derived by assuming a triangular profile with a FWHM of $20 \AA$. Line intensities for V1016 Cyg were derived from Ahern (1978). For both systems, the strongest 10 lines between 3500 and $7500 \AA$ are plotted. No absolutely calibrated line fluxes could be found for RR Tel in the visual range.

In Figures $4 a$ and $4 b$, we have plotted the 10 strongest emission lines between 1200 and $3200 \AA$ from Feibelman (1982), again assuming a triangular line profile with a FWHM of about $0.6 \AA$. The lines in Figure $4 c$ for RR Tel were obtained from Hayes and Nussbaumer (1986). These lines have not been corrected for reddening.

In the X-ray region, the Einstein X-ray spectra (not corrected for interstellar extinction) of Kwok and Leahy (1984) were used. The X-ray emission can be understood as thermal emission from the hot, shocked gas as the result of the interaction between the winds of the hot and cool components (Kwok and Leahy 1984; Willson et al. 1984).

Because of the variable nature of symbiotic novae, the energy distributions shown in Figure 4 can be viewed only as a general representation but not an accurate portrait at one single epoch. It is clear, however, that most of the observed flux from these symbiotic novae is in the infrared. Table 5 shows an approximate breakdown of the observed fluxes in the different wavebands.

The energetics of symbiotic novae can be summarized as follows. One can assume that the dust envelope is heated primarily by the cool star (see $\S$ II) and the luminosity of the cool component is emitted entirely in the infrared (photosphere + dust). The X-ray flux is derived from the mechanical energy of the wind of the hot star, which represents a small $(<5 \%)$ fraction of the hot star's total luminosity. The nebular emissions (UV + visible + radio) derive their energy from the Lyman-continuum flux from the star. If the nebula is not ionization-bounded, then part of the Lyman continuum may be escaping. Without observations in the far-ultraviolet, this fraction cannot be directly determined. However, from Table 5, we see that the nebular component contributes less than $10 \%$ of the total observed luminosity. After correction for reddening, this value may go up by a factor of 2 or 3 .

One can also determine whether there is significant leakage of flux in the ultraviolet by performing calculations based on the case $\mathbf{B}$ approximation. In a gas nebula that is completely optically thick in the Lyman continuum, the total luminosity can be related to the optically thin radio flux $\left(F_{v}\right)$ by the following formula:

$$
L_{*}=C F_{v}(\mathrm{mJy})(D / \mathrm{kpc})^{2} L_{\odot}
$$

TABLE 5

Percentage Contributions to Total Observed Flux

\begin{tabular}{|c|c|c|c|}
\hline Region & HM Sge & V1016 & RR Tel \\
\hline $\mathrm{X}$-ray $\ldots \ldots \ldots \ldots$ & 4. $(-3)$ & 1. $(-3)$ & 8. $(-4)$ \\
\hline UV continuum & 0.10 & 0.76 & 1.2 \\
\hline UV lines $\ldots \ldots \ldots . .$. & 0.08 & 0.69 & 1.9 \\
\hline Visible continuum & 0.73 & 0.78 & $\ldots^{\mathrm{a}}$ \\
\hline Visible lines ......... & 0.45 & 0.96 & $\ldots{ }^{\mathrm{a}}$ \\
\hline 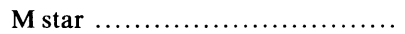 & 61.0 & 60.9 & 86.3 \\
\hline Dust envelope $\ldots \ldots \ldots \ldots \ldots \ldots$ & 38.0 & 36.0 & 10.6 \\
\hline 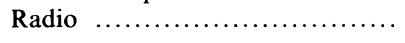 & 6. $(-5)$ & 2. $(-3)$ & 1. $(-5)$ \\
\hline Total flux (ergs $\left.\mathrm{cm}^{-2} \mathrm{~s}^{-1}\right)$ & $9.5(-8)$ & $3.8(-8)$ & $6.2(-8)$ \\
\hline
\end{tabular}

a No absolutely calibrated visible data could be found for RR Tel. 


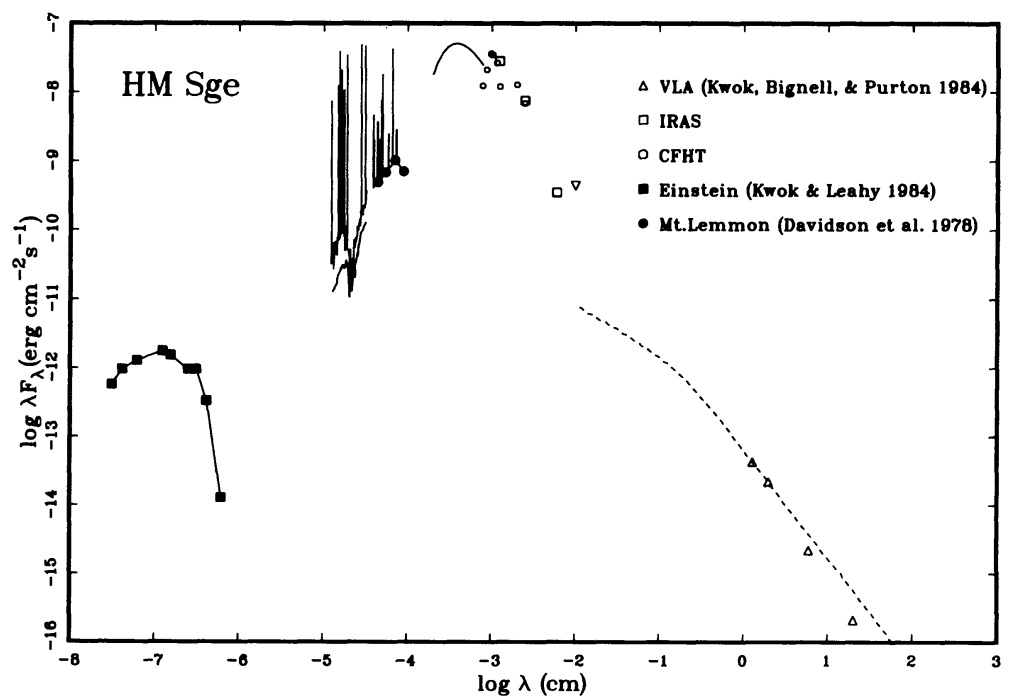

FIG. $4 a$

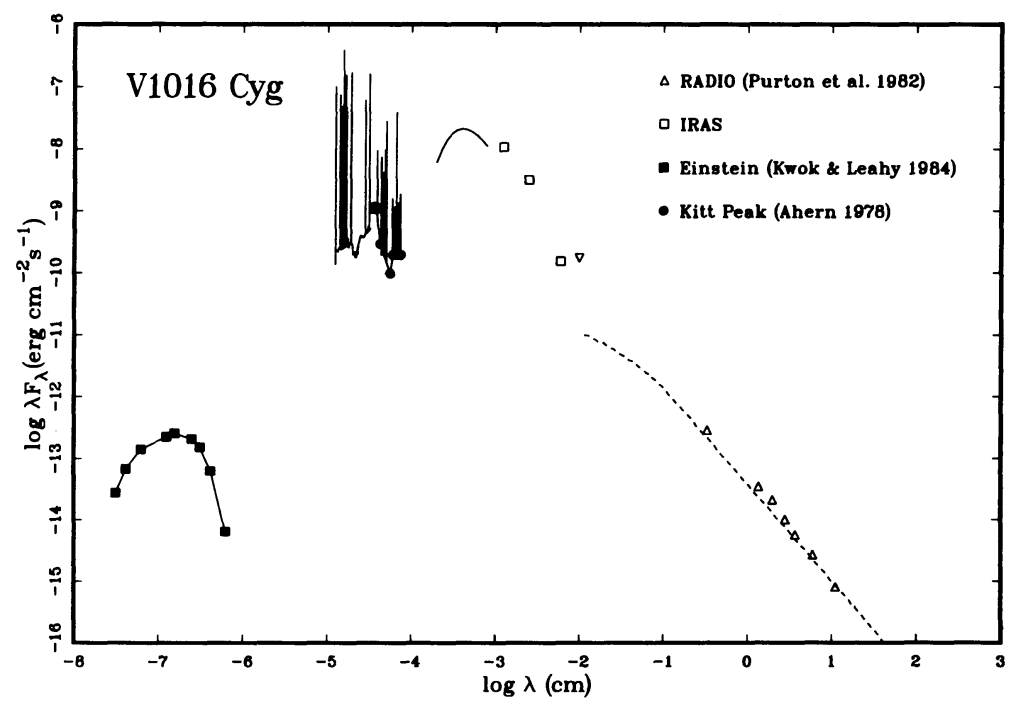

FIG. $4 b$

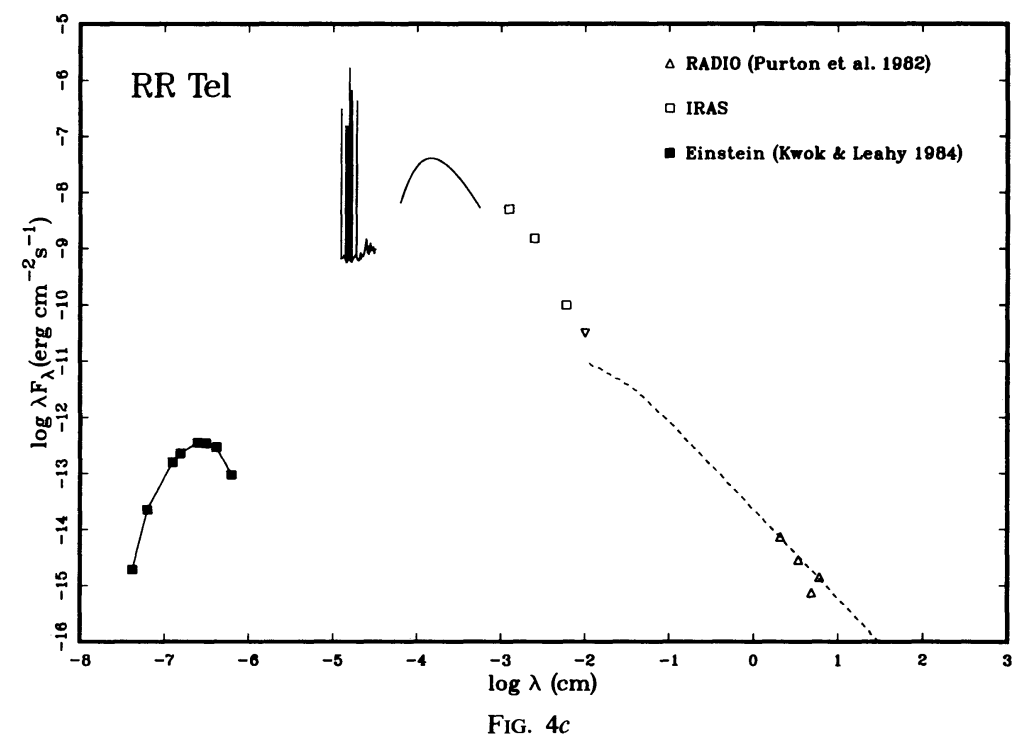

FIG. 4. - The energy distributions of (a) HM Sge, $(b)$ V1016 Cyg, and (c) RR Tel. All observational data are not corrected for interstellar extinction. The solid lines

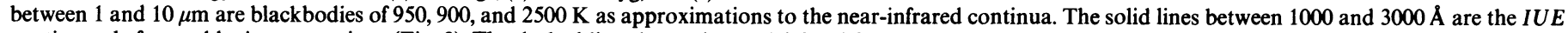

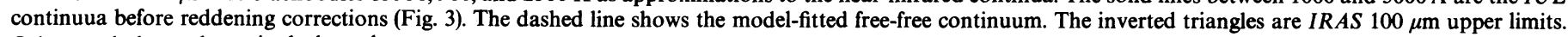
Other symbols are shown in the legend. 
where $C$ has values of $0.97-1.45$ for stellar temperatures of 40,000 to 100,000 K (Zijlstra and Pottasch 1989; Zijlstra 1989). For all three symbiotic novae, the bolometric luminosities estimated by equation (1) are $\sim 500 L_{\odot}$, or approximately onetenth of the luminosity of the cool component. This implies that the temperature of the hot component is significantly higher than $100,000 \mathrm{~K}$ and the value of $\mathrm{C}$ used is to low; or the case $\mathrm{B}$ approximation is not a good one for symbiotic novae. This conclusion would not be drastically different even if the dust envelope were completely heated by the hot component, for the cool star photospheric emission represents more than $60 \%$ of the total observed flux.

From evolutionary considerations, the hot component must have a higher progenitor mass than the cool component and therefore should be more luminous. If this is the case, then a significant amount of energy from the hot component must be escaping in the far ultraviolet.

An alternative to the density-bound hypothesis is that the luminosity of the hot component has declined significantly since the outburst. Instead of being on the horizontal track occupied by hydrogen-burning central stars of planetary nebulae, these stars may have less than $10^{-4} M_{\odot}$ in the hydrogen envelope and are evolving along the cooling track (Schönberner 1983). We find this explanation less plausible because the amount of accumulated accreted matter needed to initiate burning should last for more than a few decades (unless the white dwarf mass is very high; Kenyon and Truran 1983).

\section{DISCUSSION}

In $\S$ II and III we have shown that most of the observed flux from symbiotic novae originates from the cool component, and the infrared spectra can be understood by the combination of a dust envelope heated by the cool star and the reddened photosphere of the cool star itself. We have also suggested that the energy sources are clearly separated: the hot star is responsible for the ionized gas nebula which emits in the X-ray, UV, visible, and radio, and the cool component heats the dust envelope which emits in the infrared. The fact that only a small fraction of the total flux is emitted by the gas nebula suggests that the nebula is unlikely to be ionization-bounded. This could be the result of the geometry of the system.

The geometric structure of symbiotic novae has been studied in detail by Girard and Willson (1987). Their dynamical model is based on the observation that both the hot and cool components have winds, and the evolution of the density structure of the circumstellar envelope is likely to be shaped by the interaction of the two winds (Kwok 1982). This departure from spherical symmetry can also affect the ionization structure of the system. Although the orbital periods of classical $S$ type symbiotic systems seem to be in the 1-3 yr range (Kenyon and Webbink 1984; Garcia and Kenyon 1988), there are some suggestions that symbiotic novae might have significantly longer $(P>20 \mathrm{yr}$ ) orbital periods (Willson, Garravich, and Mattei 1981; Wallerstein et al. 1984; Whitelock 1987). If the binary separation is a large fraction of the size of the circumstellar envelope, then one can imagine that the system will be ionization-bounded in the volume between the two stars, but density-bounded away from the cool component (Seaquist, Taylor, and Button 1984). The conclusion in $\S$ III of UV leakage could be the result of such geometry. Alternatively, the circumstellar envelope of the cool component may not be spherically symmetric. The high- $\left(00^{\prime \prime 06}\right)$ resolution map of HM Sge shows that it has a "bipolar" structure (Kwok, Bignell, and Purton 1984), probably as the result of a prolate ellipsoidal shell viewed at an angle. If the mass-loss process concentrates more on the equatorial plane, then the nebula may be ionization-bounded in the equatorial plane but densitybounded in the polar directions.

Another interesting aspect of the nature of symbiotic novae is the shape of their visible light curves. Unlike classical novae, the visual maximum of symbiotic novae can last for decades (Viotti 1988). While visible light of classical novae is dominated by continuum emission from the white-dwarf ejecta, we can see from Figure 4 that the visible light from symbiotic novae is dominated by nebular line emission. If the nebula is not ionization-bounded, then the visual brightness will not be very sensitive to small changes in the luminosity or temperature of the hot component, so long as its temperature remains $>50,000 \mathrm{~K}$. Without observations in the far-ultraviolet, the evolution of the hot component may be difficult to detect.

There is, however, evidence that the hot component has been changing. The temperature of the hot component of HM Sge inferred from the emission lines changed from $\sim 50,000 \mathrm{~K}$ after the outburst to $\sim 160,000 \mathrm{~K}$ in 1984 (Stauffer 1984; Mueller and Nussbaumer 1985). This increase in temperature may have continued as late as 1989 , when it is estimated to be $170,000 \mathrm{~K}$ (Nussbaumer and Vogel 1990). The high-velocity wind also ceased in 1978 (Stauffer 1984). These two pieces of observational data are in fact consistent with each other, for the massloss rate from the hot component is expected to decrease as the stars evolve to higher temperatures if the mass loss is driven by radiation on resonance lines (Kudritzki and Méndez 1989).

The difference in the visual light curves between classical and symbiotic novae can therefore be traced down to the existence of circumstellar envelopes before the outburst in symbiotic novae. In classical novae, the ejection from the hot component creates a large optically thick (continuum) surface, and the resultant low $(\sim 10,000 \mathrm{~K})$ effective temperature of this surface causes the light to peak in the visible range. As the ejection rate decreases, the surface shrinks, the effective temperature increases, and the visible light declines. For symbiotic novae, the visual outburst is the result of the ionization of the preexisting AGB envelope and the emergence of emission lines (Kwok and Purton 1979). Since the ejecta from the hot component represents only a small fraction of the total mass, the visual light will remain relatively constant as long as the nebula remains ionized.

\section{CONCLUSIONS}

The IRAS LRS of the three symbiotic novae discussed here all show prominent 10 and $18 \mu \mathrm{m}$ silicate emission features, suggesting that the cool components of these systems are similar to mass-losing Mira variables on the AGB. Dust continuum radiative transfer model fitting to the observed infrared spectra suggest that the dust envelopes are detached from the photospheres of the cool stars. Unlike normal Mira variables, the inner radii of the dust envelopes are located 10-60 stellar radii from the star, probably as the result of a "snowplow" effect from the fast ejection from the hot component. The derived mass-loss rates are typical of those of evolved Mira variables and are also consistent with the rates estimated from the radio continuum emission.

While the silicate features suggest that the cool components of these systems are oxygen-rich, the $2200 \AA$ absorption feature, usually attributed to carbon-based grains, is prominent in the ultraviolet spectra of the objects. We find that the 
$2200 \AA$ absorption feature has decreased in strength in HM Sge after 1978, suggesting that this feature is created during the white dwarf ejection process and is not interstellar in origin. This association is consistent with the termination of the white dwarf ejection in 1978 as witnessed in the visible spectrum.

All three symbiotic novae are active in every part of the electromagnetic spectrum. While the mid- and far-infrared spectrum is dominated by circumstellar dust emission, the near-infrared continuum is primarily due to the reddened photosphere of the cool star. In the visible, ultraviolet, and radio regions, line and continuum emission from the ionized nebula dominate. The $\mathrm{X}$-ray continuum is probably created by dynamical heating as the result of the interaction between ejection from the hot component and the nebula. Analysis of the energy budget of the systems suggests that most of the observed flux is due to the cool component, and the observed contributions from the ionized nebulae fall short of the expected Lyman-continuum fluxes of the hot component. This suggests that a significant fraction of the energy of the hot star is probably escaping in the far-ultraviolet. From the observed energy distributions we estimate that the lower limits to the luminosities are 2970,1190 , and $1940 L_{\odot}(D / k p c)^{2}$ for HM Sge,
V1016 Cyg, and RR Tel respectively. The actual luminosities may be higher due to the leakage of UV photons. The densitybounded nature of the nebulae could be related to the bipolar geometry of the systems.

The existence of the AGB envelope before the ignition of the white dwarf is responsible for the long duration of the visual maximum in symbiotic novae. The visual magnitude of symbiotic novae is dominated by line emission from the AGB envelope, and not by continuum emission as in the case of classical novae. The origin of the visual outburst is also different from that of classical novae in that it is caused by the ionization of the AGB envelope and is not due to a sudden ejection of matter by the hot component.

We thank K. M. Volk for helpful discussions and Paul Wesselius for providing us with the LRS database. We also thank E. Brugel and the staff of the University of Colorado IUE Regional Data Analysis Facility for assistance in the processing of IUE data. B. J. Hrivnak and R. T. Boreiko assisted in the CFHT observations. This work is supported by a grant from the Natural Sciences and Engineering Research Council of Canada.
Ahern, F. J. 1978, Ap. J., 223, 901.

Allen, D. A. 1980, M.N.R.A.S., $190,75$.

1981, M.N.R.A.S., 197, 789 .

Anandarao, B. G., Taylor, A. R., and Pottasch, S. R. 1988, Astr. Ap., 203, 361.

Arkhipova, V. P., and Dokuchaeva, O. D. 1978, Soviet Astr. Letters, 4, 48.

Baratta, G. B., Cassatella, A., and Viotti, R. 1974, Ap. J., 187, 651.

Blair, W. P., Stencel, R. E., Shaviv, G., and Feibelman, W. A. 1981, Astr. Ap., 99, 73.

Ciatti, F., Mammano, A., and Vittone, A. 1978, Astr. Ap., 68, 251

Davidson, K., Humphreys, R. M., and Merrill, K. M. 1978, Ap. J., 220, 239.

Feast, M. W., and Whitelock, P. A. 1987, in Late Stages of Stellar Evolution, ed.

S. Kwok and S. R. Pottasch (Dordrecht: Reidel), p. 33.

Feibelman, W. A. 1982, Ap. J., 238, 548.

Garcia, M. R., and Kenyon, S. J. 1988, in IAU Colloquium 103, The Symbiotic

Phenomenon, ed. J. Mikolajewska, M. Friedjung, S. J. Kenyon, and R. Viotti (Dordrecht: Kluwer), p. 27.

Girard, T., and Willson, L. A. 1987, Astr. Ap., 183, 247.

Hayes, M. A., and Nussbaumer, H. 1986, Astr. Ap., 161, 287.

Kenyon, S. J., Fernandez-Castro, T., and Stencel, R. E. 1986, A.J., 92, 1118.

. 1988, A.J., 95, 1817 .

Kenyon, S. J., and Truran, J. W. 1983, Ap. J., 273, 280.

Kenyon, S. J., and Webbink, R. F. 1984, Ap. J., 279, 252.

Kudritzki, R. P., and Méndez, R. H. 1989, in IAU Symposium 131, Planetary Nebulae, ed. S. Torres-Peimbert (Dordrecht: Kulwer), p. 273.

Kwok, S. 1982, in IAU Colloquium 70, The Nature of Symbiotic Stars, ed. M. Friedjung and R. Viotti (Dordrecht: Reidel), p. 209.

. 1988, in IAU Colloquium 103, The Symbiotic Fhenomenon, ed. J.

Mikolajewska, M. Friedjung, S. J. Kenyon, and R. Viotti (Dordrecht: Kluwer), p. 129

Kwok, S., Bignell, R. C., and Purton, C. R. 1984, Ap. J., 279, 188.

Kwok, S., and Leahy, D. A. 1984, Ap. J., 283, 675.

Kwok, S., and Purton, C. R. 1979, Ap. J., 229, 187.

Leung, C. M. 1975, Ap. J., 199, 340.

.1976, Ap.J., 209, 75.

Mueller, B. E. A., and Nussbaumer, H. 1985, Astr. Ap., 145, 144.

Nussbaumer, H., and Schild, H. 1981, Astr. Ap., 101, 118.

\section{REFERENCES}

Nussbaumer, H., and Vogel, M. 1990, Astr. Ap., 236, 117.

O’Dell, C. R. 1967, Ap. J., 149, 373.

Olnon, F. M. 1975, Astr. Ap., 39, 217

Paczyński, B., and Rudak, B. 1980, Astr. Ap., $82,349$.

Penston, M. V., et al. 1983, M.N.R.A.S., 202, 833.

Puetter, R. C., Russell, R. W., Soifer, B. T., and Willner, S. P. 1978, Ap. J. (Letters), 223, L93.

Purton, C. R., Feldman, P. A., Marsh, K. A., Allen, D. A., and Wright, A. E. 1982, M.N.R.A.S., 198, 321 .

Sahade, J., Brandi, E., and Fontenla, J. M. 1984, Astr. Ap. Suppl., 56, 17.

Savage, B. D., and Mathis, J. S. 1979, Ann. Rev. Astr. Ap., 17, 73 .

Seaquist, E. R., Taylor, A. R., and Button, S. 1984, Ap. J., 284, 202.

Schönberner, D. 1983, Ap. J., 272, 708.

Stauffer, J. R. 1984, Ap. J., 280, 695 .

Swings, J. P. 1981, Astr. Ap. Suppl., 43, 331.

Taranova, O. G., and Yudin, B. F., 1983, Astr. Ap., 117, 209.

Thronson, H., and Harvey, P. M. 1981, Ap. J., 248, 584.

Viotti, R. 1988, in IAU Colloquium 103, The Symbiotic Phenomenon ed. J. Mikolajewska, M. Friedjung, S. J. Kenyon, and R. Viotti (Dordrecht: Kluwer), p. 269

Volk, K., and Cohen, M. 1989, A.J., 98, 1918.

Volk, K., and Kwok, S. 1987, Ap. J., 315, 654

. 1988, Ap. J., 331, 435.

Wallerstein, G., Willson, L. A., Salzer, J., and Brugel, E. 1984, Astr. Ap., 133, 137.

Whitelock, P. 1987, Pub. A.S.P., 99, 573

1988, in IAU Colloquium 103, The Symbiotic Phenomenon, ed. J. Mikolajewska, M. Friedjung, S. Kenyon, and R. Viotti (Dordrecht: Kluwer),

p. 47.
Willson, L. A., Garavich, P., and Mattei, J. A. 1981, Inf. Bull. Var. Stars, No. 1961.

Willson, L. A., Wallerstein, G., Brugel, E., and Stencel, R. E. 1984, Astr. Ap., $133,154$.

Wright, A. E., and Barlow, M. J. 1975, M.N.R.A.S., 170, 41

Zijlstra, A. A. 1989, Ph.D. thesis, University of Groningen.

Zijlstra, A. A., and Pottasch, S. R. 1989, Astr. Ap., 216, 245.

G. L. BRYAN: Department of Astronomy, University of Illinois, Urbana, IL 61801

S. KwoK: Department of Physics and Astronomy, University of Calgary, Calgary, Alberta, Canada T2N 1N4 